\title{
Pré-Despacho de um Sistema Hidrotérmico com Manobras e Restrições de Rampa
}

\author{
S. M. S. CARVALHO ${ }^{*}$ e M. V. COELHO ${ }^{2}$ \\ Recebido em 22 de novembro de 2018 / Aceito em 16 de julho de 2019
}

\begin{abstract}
RESUMO. Os métodos de pontos interiores primais-duais foram utilizados para o problema de minimização do custo de operação do pré-despacho DC de um sistema de potência hidroelétrico e hidrotérmico onde ocorrem adicionalmente manobras programadas e restrições de rampa. Com a variação da demanda, se faz necessário realizar algumas manobras programadas para manutenção de geradores e para adaptar a rede de transmissão a esta carga possibilitando atendê-la com eficiência e mantendo o sistema estável. As usinas termoelétricas, por sua vez, apresentam restrições de rampa, pois necessitam de um determinado tempo tanto para aumentar quanto para reduzir sua produção de energia. A consideração de manobras e restrições adicionais aproxima o modelo do problema de pré-despacho do sistema brasileiro de acordo com o Operador Nacional do Sistema.
\end{abstract}

Palavras-chave: métodos de pontos interiores, restrições de rampa, manobras programadas.

\section{INTRODUÇÃO}

Desde o surgimento dos métodos de pontos interiores para programação linear, códigos computacionais baseados nessas idéias vêm se apresentando como alternativas eficientes para solução de problemas de grande porte $[1,3,8,11,13,23]$.

Uma linha de pesquisa importante considera classes específicas de problemas e explora as particularidades da estrutura matricial com o objetivo de obter implementações ainda mais eficientes. Esta exploração da estrutura pode levar a métodos de solução muito diversificados. Assim, dependendo do método e da classe de problemas, a melhor opção pode ser a utilização de métodos iterativos [25], decomposição $L U$ ou Cholesky [12, 20,21, 22], uma combinação entre decomposição e métodos iterativos [7], ou mesmo a redução do problema de tal forma que

\footnotetext{
*Autor correspondente: Silvia M.S. Carvalho - E-mail: silviamsc@ufscar.br - https://orcid.org/0000-0001-9519-9339

${ }^{1}$ Departamento de Física, Química e Matemática, DFQM, Universidade Federal de São Carlos, Rodovia João Leme Santos, 13566-590, Sorocaba-SP, Brasil E-mail: silviamsc@ufscar.br

2 Instituto de Ciência e Tecnologia - ICT, Rua Gabriel Monteiro da Silva, 700, 37130-001 Alfenas-MG E-mail: mayk@gmail.com
} 
a esparsidade apareça apenas implicitamente $[17,18]$.Todas estas aplicações tem como resultado a obtenção de implementações mais eficientes que a utilização de métodos genéricos de otimização.

$\mathrm{Na}$ área de sistemas de potência o advento dos métodos de pontos interiores trouxe à tona uma nova linha de pesquisa. Estes métodos são reconhecidos atualmente por sua robustez [10,15,24], principalmente devido ao tratamento eficiente de desigualdades.

\section{RESTRIÇÕES DE RAMPA}

Em função do grande potencial hídrico, o Brasil utiliza a energia termoelétrica de forma estratégica. Esse uso ocorre normalmente quando há diminuição de água, provocada pela carência de chuvas, nas represas que abastecem as usinas hidrelétricas. Geralmente, as termoelétricas geram entre $15 \%$ e $20 \%$ da energia elétrica consumida no país. A energia termelétrica é um tipo de energia produzida a partir de uma fonte de calor. Esta fonte pode ser obtida via a queima de carvão, óleo combustível, gás natural, combustíveis nucleares, etc.

As vantagens das usinas termoelétricas é que em comparação com usinas hidrelétricas, são mais rápidas para serem construídas, podendo assim suprir carências de energia de forma mais agil, podem também ser instaladas em locais próximos às regiões de consumo, reduzindo o custo com torres e linhas de transmissão e são altamente eficientes. Se por um lado o uso de usinas termelétricas apresenta uma ótima eficiência energética, com alta capacidade de produção anual integral (diferentemente das usinas hidroelétricas que dependem das cheias dos rios para seu funcionamento); por outro lado há de se ressaltar também que o modelo de produção das usinas termelétricas adotado no Brasil é feito predominantemente da queima de combustíveis fosseis, sendo apenas um terço das usinas termelétricas do país à base de biomassa, tendo a cana de açúcar como o principal combustível. O que ressalta a importância de se investir em tecnologias para a produção eficiente de energia.

No sistema elétrico existe uma variação de carga entre intervalos de tempos distintos, na prática, sabe-se que a tomada de carga se dá sob a forma de uma rampa cuja inclinação é ditada pelos limites físicos das unidades geradoras. A consideração do efeito de rampa implica no uso de restrições intertemporais que estabelecem a interrelação entre variáveis associadas a diversos intervalos de tempo do horizonte de operação considerado. É comum na operação limitar a rampa, e essa restrição pode ser da própria máquina, que possui uma limitação de tomada e de alívio de carga, ou por outras razões, tais como restrições de navegação, saneamento e ambiental.

As usinas termoelétricas apresentam restrições de rampa, pois necessitam de um determinado tempo tanto para aumentar quanto para reduzir sua produção de energia. A restrição de rampa acopla o nível de geração de uma unidade termoelétrica entre dois períodos consecutivos da programação, onde não é possível admitir variações abruptas da potência gerada nesse intervalo de tempo. 
Nesse trabalho, as restrições de rampa referem-se à capacidade máxima de acréscimo e ou de redução de geração da termoelétrica, em relação à hora anterior dado em $M W h$. Esta restrição pode ser escrita como [19]:

$$
p_{i+1}-d_{i} \leq p_{i} \leq p_{i+1}+d_{i},
$$

para $i=1,2, \ldots t-1$, onde $d_{i}$ é o vetor que representa a variação de energia permitida no intervalo de tempo $i$ em cada usina termoelétrica.

Maiores detalhes sobre a metodologia dos métodos de pontos interiores e sua aplicação no prédespacho com manobras pode ser visto em $[5,14]$.

\section{MANOBRAS PROGRAMADAS}

As manobras são realizadas na tentativa de se adaptar a rede de transmissão a variação da carga (variação da demanda de energia) ao longo do dia. Na maior parte dos intervalos de tempo não são realizadas manobras no sistema elétrico brasileiro, fazendo com que a rede de transmissão raramente se altere de um intervalo para outro, normalmente são realizadas de quatro a seis manobras por dia. As mudanças aqui consideradas serão chamadas de manobras preventivas, ou seja, aquelas alterações que ocorrem na rede para que seja feita manutenções evitando assim interrupções de energia.

O Centro de Operação do Sistema (COS) é o responsável por executar, autorizar e supervisionar as manobras e serviços programados ou emergenciais do sistema elétrico de transmissão, realizar o monitoramento do mesmo, bem como atuar efetivamente no restabelecimento do sistema elétrico em caso de contingências simples e generalizadas. Tais atividades, executadas em tempo real, abrangem o conhecimento da situação e a orientação na execução de manobras necessárias, visando assegurar a integridade de pessoas e instalações, garantindo a confiabilidade do sistema e a continuidade e qualidade do fornecimento.

Três tipos de manobras serão consideradas nesse trabalho:

- manobras de linhas;

- manobras de barras;

- manobras simultâneas de linhas e barras.

\subsection{Manobras de Linhas}

Quando uma manobra de linha é realizada, ou seja, um ramo é retirado do sistema, a topologia da rede é modificada. Algebricamente, uma coluna da matriz de incidência, referente ao ramo manobrado, e uma linha da matriz de reatância são retiradas [6].

Para eficiência computacional optou-se por fazer manobras somente nos arcos adicionais da árvore geradora. Sem perda de generalidade, é possível utilizar uma heurística para construção de uma árvore geradora, de forma a garantir que os ramos a serem manobrados sempre pertençam a co-árvore [20]. 


\subsection{Manobras de Barras}

Uma manobra de barra ocorre quando geradores ou cargas são desligados do sistema. As cargas têm variações nem sempre previsíveis com o tempo, fator que pode dificultar a modelagem do sistema.

Diferentemente das manobras de linhas, nas manobras de barras, não se pode optar por ramos pertencentes ou não a árvore geradora, pois obviamente ao desligar uma barra, linhas da árvore geradora e de seus arcos adicionais devem ser desligados. Para evitar danos ao sistema, escolheu-se fazer manobras somente nas barras com grau menor que dois (somente um arco da árvore tocando nele). Novamente é fácil alterar a heurística em [20] para obter árvores com essas características. Supõe-se que $n$-arcos incidem na barra a ser manobradas, $\log$ o $n$-linhas e $n$-colunas das matrizes de reatância e incidência respectivamente devem ser retirados.

\section{FORMULAÇÃO DO PROBLEMA DE PRÉ-DESPACHO COM MANOBRAS E RESTRIÇÕES DE RAMPA}

Como já destacado anteriormente, o pré-despacho de um sistema elétrico é um problema operacional de curto-prazo, que pode ser formulado com a minimização de uma função quadrática com variáveis separáveis, onde o objetivo a ser minimizado considerando os custos de geração. As leis de Kirchoff caracterizam restriçoes do problema, um conjunto de equações matriciais que se modificam ao longo de horizontes de estudo, em consequência das manobras programadas.

O custo de geração associado às termoelétricas também é uma função quadrática independente para cada gerador. Vale ressaltar que os métodos de pontos interiores para problemas com esta característica apresentam desempenho similar ao obtido para problemas lineares. Em particular o esforço por iteração é virtualmente o mesmo em ambas as situações [26,27].

Nesse momento, será desenvolvido o método de pontos interiores primal-dual para o prédespacho DC de um sistema elétrico onde ocorrem adicionalmente manobras programadas e restrições de rampa.

O problema de fluxo de carga ótimo DC foi estudado em [20,21] e a estrutura matricial do modelo explorada resultando em uma implementação bastante rápida e robusta. Este trabalho por sua vez foi estendido em [22] para o problema de pré-despacho sem a consideração de manobras programadas e restrições de rampa ao longo do dia. 


\subsection{O Modelo Estático}

Primeiramente, apresentaremos o modelo em um intervalo de tempo do fluxo de potência ótimo ativo [4]:

$$
\begin{array}{cc}
\text { minimizar } & \alpha f^{t} R f+\beta\left[p^{T} Q p+c^{T} p\right] \\
\text { sujeito a } & A f=E p-l \\
& X f=0 \\
& p-d \leq p \leq p+d \\
& f^{\text {min }} \leq f \leq f^{\max } \\
& p^{\text {min }} \leq p \leq p^{\max }
\end{array}
$$

onde:

$f$ representa o vetor de fluxo de potência ativa;

$p$ representa o vetor de geração de potência ativa;

$R$ representa a matriz diagonal das resistências das linhas;

$Q$ representa a matriz de componente quadrática do custo de geração;

$c$ é o vetor associado ao termo linear dos custos de geração;

$A$ representa a matriz de incidência da rede de transmissão;

$E$ representa a matriz formadas de colunas da identidade correspondendo às barras de geração;

$X$ representa a matriz de reatância da rede de transmissão;

$l$ representa o vetor de demandas de potência ativa;

$d$ é o vetor que representa a variação de energia;

$f^{\text {max }}$ e $f^{\text {min }}$ são os limites de fluxo de potência ativa;

$p^{\max }$ e $p^{\text {min }}$ são os limites de geração de energia hidráulica;

$\alpha$ e $\beta$ são ponderações dos objetivos a minimizar.

O sistema de transmissão é representado por um fluxo de carga DC (i.e., fluxo de carga em corrente contínua) com limites no fluxo de carga ativo. Para que as variáveis de geração $(p)$ e de transmissão $(f)$ possam ser expressas simultaneamente no modelo, as leis de Kirchhoff são apresentadas separadamente [4]. As equações em (4.2) e (4.3) representam as leis de Kirchhoff para nós e circuitos respectivamente. Portanto, o conjunto de restrições para este problema é linear onde essas equações representam a rede de geração/transmissão. A equação (4.4) representa as restrições de rampa das usinas termoelétricas, já as equações (4.5) e (4.6)representam as capacidades de geração e transmissão do sistema. Este modelo é mais eficiente que o modelo baseado no princípio do mínimo esforço [16].

\subsection{O Modelo Dinâmico}

A representação do problema descrita anteriormente corresponde a um único intervalo de tempo da operação. Para estender a formulação (4.1-4.6), é necessário considerar este problema para 
cada intervalo de tempo, considerando que com a inserção de manobras programadas a rede de transmissão pode variar de um intervalo de tempo para outro.

A seguinte equação representa o atendimento das metas para cada usina hidroelétrica no período de 24 horas:

$$
\sum_{i=1}^{t} p^{k}=q
$$

onde $q$ representa a meta de geração de energia das hidroelétricas para o horizonte em estudo, estabelecida pelo planejamento de longo prazo, $p_{i}$ representa a geração de potência ativa no intervalo de tempo $i$ e o número total de intervalos é dado por $t$.

Quando existem manobras do intervalo de tempo $i$ para o intervalo seguinte a configuração topológica da rede se altera e as matrizes de incidência nó arco $A^{i}$ e de reatância $X^{i}$ ficam distintas das matrizes $A^{i+1}$ e $X^{i+1}$, respectivamente. As matrizes $R, Q$ e $E$ também podem se alterar de um intervalo de tempo para o seguinte.

Considerando agora o modelo com $t$ intervalos de tempo. O Problema de pré-despacho com manobras e restrições de rampa, pode ser simplificado utilizando as seguintes mudanças de variáveis [21]:

$$
\begin{aligned}
& \widetilde{f}^{k}=f^{k}-f^{\min } \\
& \widetilde{p}^{k}=p^{k}-p^{\text {min }}
\end{aligned}
$$

Com essas modificações. o problema fica como descrito em 4.7, omitiremos os tils para simplificar a notação.

$$
\begin{array}{cc}
\text { Min } & \frac{\alpha}{2} \sum_{k=1}^{t}\left[\left(f^{k}\right)^{T} R^{k} f^{k}+c_{f}^{T} f^{k}\right]+\frac{\beta}{2} \sum_{k=1}^{t}\left[\left(p^{k}\right)^{T} Q^{k}\left(p^{k}\right)+c_{p}^{T} p^{k}\right] \\
A^{k} f^{k}-E^{k} P^{k}=l^{k} & \\
\text { sujeito a } & X^{k} f^{k}=0 \\
p^{k+1}-d^{k} \leq p^{k} \leq p^{k+1}+d^{k} \\
\sum_{i=1}^{t} p^{k}=q .
\end{array}
$$

É fácil perceber que as restrições de fluxo potência ativa e segurança se repetem para cada intervalo de tempo e apenas se acoplam através das restrições adicionais de metas.

O problema dual pode ser escrito como:

$$
\begin{gathered}
\max \sum_{k=1}^{t}\left[\left(\widehat{d}^{k}\right)^{T} y_{\widetilde{f}}^{k}-\left(\widetilde{f}^{\max }\right)^{T} w_{\widetilde{f}}^{k}-\left(\widetilde{p}^{\max }\right)^{T} w_{\widetilde{p}}^{k}+(\widetilde{q})^{T} y_{a}-\frac{\alpha}{2}\left[\left(\widetilde{f}^{k}\right)^{T} R \widetilde{f}^{k}\right]-\frac{\beta}{2} \sum_{k=1}^{t}\left[\left(\widetilde{p}^{k}\right)^{T} Q \widetilde{p}^{k}\right]\right. \\
\text { s.a } B^{T} y_{\widetilde{f}}^{k}-w_{\widetilde{f}}^{k}-R \widetilde{f}^{k}+z_{\widetilde{f}}^{k}=c_{\widetilde{f}} \forall k=1, \ldots, t \\
-\widehat{E}^{T} y_{\widetilde{f}}^{k}-w_{\widetilde{p}}^{k}+y_{a}-Q \widetilde{p}^{k}+z_{\widetilde{p}}^{k}=c_{\widetilde{p}} \quad \forall k=1, \ldots, t \\
y_{4}^{k}+w_{3}^{k}-z_{3}^{k}=0 \quad \forall k=1, \ldots, t \\
\left(z_{\widetilde{f}}^{k}, w_{\widetilde{f}}^{k}, z_{\widetilde{p}}^{k}, z_{3}^{k}, w_{\widetilde{p}}^{k}, w_{3}^{k}\right) \geq 0, \quad y_{\widetilde{f}}^{k}, y_{a} \text { livres. }
\end{gathered}
$$




\section{ESTUDO DA ESTRUTURA MATRICIAL}

Supõe-se que ocorram $i$ manobras previamente programadas ao longo de $t$ intervalos de tempo, onde cada intervalo de tempo corresponde a um período de 1 ou $1 / 2$ hora. $O$ número de manobras $i$ é normalmente pequeno no sistema brasileiro, variando de zero a seis.

A matriz $B$, formada pelas linhas justapostas da matriz de incidência e reatância, não é mais constante ao longo dos $t$ intervalos de tempo. Cada vez que uma manobra é realizada, uma linha e uma coluna da matriz $B$ são retiradas (inseridas). No caso de existir mais de uma manobra no mesmo intervalo de tempo, um número maior de linhas e colunas da matriz $B$ são retiradas (inseridas).

Quando considerarmos um sistema com manobras em diferentes intervalos de tempo, utilizaremos a seguinte notação:

$$
B^{k}=\left[\begin{array}{l}
A^{k} \\
X^{k}
\end{array}\right] .
$$

onde

$$
k=1,2, \ldots, t \text {. }
$$

Assim, temos descrita a topologia da rede utilizada. Por simplicidade de notação iremos omitir os índices.

Como a dimensão da matriz $B$ pode se modificar a cada manobra, deve-se ajustar o sistema a essas mudanças. Ou seja, para a realização do produto e somatório com $B$, as dimensões e estruturas das outras matrizes envolvidas no sistema (decorrentes das características de $B$ ) são modificadas.

A matriz $B$ pode ser decomposta como:

$$
B=\left[\begin{array}{l}
A \\
X
\end{array}\right] .
$$

De forma mais detalhada:

$$
B=\left[\begin{array}{cc}
T & N \\
X_{T} & X_{N}
\end{array}\right],
$$

onde:

$$
A=\left[\begin{array}{ll}
T & N
\end{array}\right]
$$

$\mathrm{e}$

$$
X=\left[\begin{array}{ll}
X_{T} & X_{N}
\end{array}\right]
$$

Com esta decomposição, as colunas da matriz de incidência $A$ estão divididas de tal forma que $T$ contém os arcos de uma árvore geradora e $N$ é formada pelos demais arcos, pertencentes, a co-árvore [2]. A matriz de reatância $X$ é particionada de forma equivalente. 
Por exemplo, se tivermos a árvore geradora aqui considerada e adicionarmos alguns arcos, formaremos o sistema a ser trabalhado como mostra a Figura 1 e cuja matriz de incidência é mostrada na Figura 3, supondo-se que façamos manobras (desligar) nos ramos pontilhados da Figura 2.

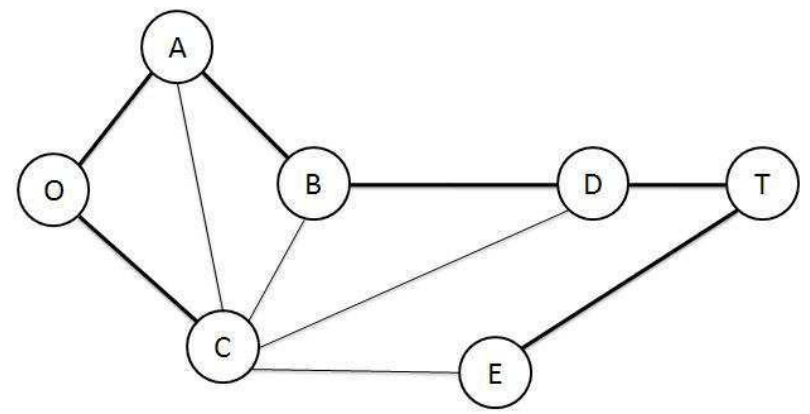

Figura 1: Árvore geradora com arcos em negrito e arcos adicionais.

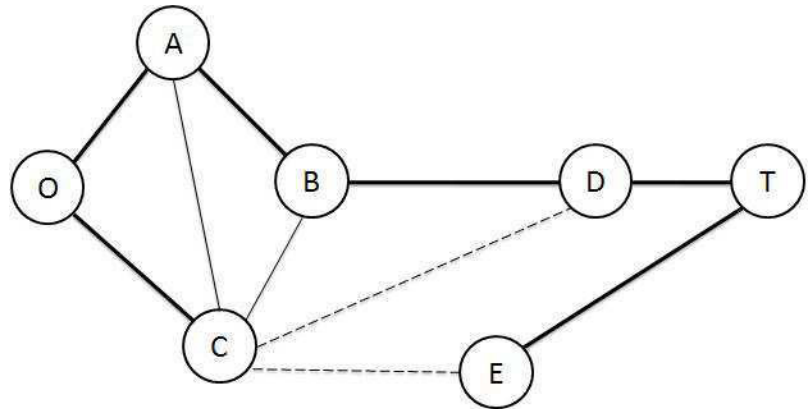

Figura 2: Ramos a serem desligados no sistema.

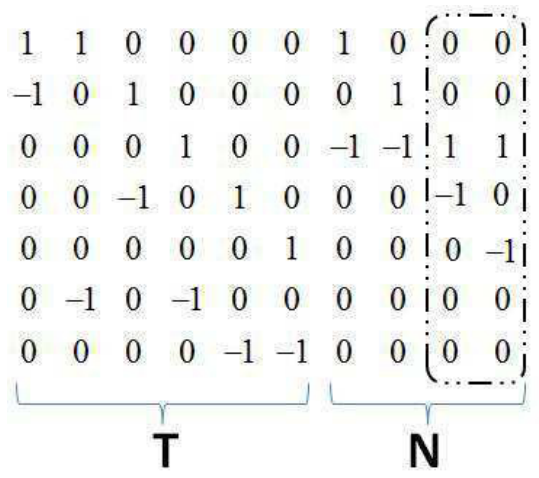

Figura 3: Colunas a serem desligadas da matriz de incidência. 


\subsection{Heurística para Construção da Árvore Geradora}

O objetivo dessa seção é mostrar a heurística utilizada para a construção das árvores geradoras utilizadas nos experimentos realizados. Neste trabalho, optou-se pela construção de uma árvore geradora com arcos adicionais. Dessa forma, é possível ter uma matriz de reatância cuja estrutura possa ser explorada com eficiência.

A matriz de reatância será denotada por $\left[\begin{array}{ll}X_{T} & X_{N}\end{array}\right]$, onde a submatriz $X_{N}$ é diagonal e representa os arcos da co-árvore. É sabido que a esparsidade da matriz de reatância depende dos circuitos adotados em sua construção [14]. A seguinte heurística busca construir uma matriz de reatância esparsa:

- É escolhida a barra com maior grau como raiz, e todos seus vizinhos como filhos;

- As barras remanescentes vizinhas da folha de maior grau são então acrescentadas na árvore;

- O procedimento é repetido até que todas as barras façam parte da árvore.

Assim, procura-se construir uma árvore com profundidade pequena. Os laços obtidos acrescentando uma linha não pertencente a árvore de cada vez formam a matriz de reatância. Em uma árvore pouco profunda, estes laços tendem a conter poucas barras, resultando em uma matriz esparsa. Observe também que cada arco adicional pertence a um único circuito, ou seja, $X_{N}$ é diagonal. Finalmente, dado que $X_{N}$ é diagonal, a matriz de reatância tem linhas linearmente independentes e, uma vez que há $n-m+1$ arcos fora da árvore, obtemos o número de equações necessário para formar $X[9]$.

As matrizes $B^{k}=L^{k} U^{k}$ podem ser decompostas antes do processo iterativo, de forma análoga ao que pode ser realizado com a matriz $B$ no caso do problema sem manobras e observe que a matriz $\left[\begin{array}{ll}B & E\end{array}\right]$ tem posto completo, logo não existem restrições redundantes no problema.

Tanto para manobras de linhas como de barras, a matriz $B^{k}$ é armazenada para cálculos posteriores da resolução de sistemas lineares do método de pontos interiores. Por questão de funcionalidade, são armazenados somente os ramos que estão ativos no sistema, ou seja, as colunas da matriz de arcos-adicionais que estão ligadas em um determinado intervalo de tempo. 


\section{RESULTADOS COMPUTACIONAIS}

Os testes realizados nesta seção usaram o ponto inicial mostrado na Equação (6.1). Este foi definido como em [22], que apresentou bons resultados em experimentos anteriores.

$$
\begin{gathered}
f^{0}=\frac{f^{\max }}{2} \\
p^{0}=\frac{p^{\text {max }}}{2} \\
y_{1}^{0}=y_{2}^{0}=y_{3}^{0}=y_{4}^{0}=0 \\
z_{1}^{0}=w_{1}^{0}=(R+I) e \\
z_{2}^{0}=w_{2}^{0}=e \\
z_{3}^{0}=w_{3}^{0}=e .
\end{gathered}
$$

Um estudo ilustrativo pode ser feito com sistema IEEE30, com 6 unidades geradoras, potência instalada de 390Mw e uma carga de 283,4Mw. A Figura 4 mostra seu diagrama unifilar.

Foram realizados testes computacionais com as seguintes configurações: Imac com MacOS Mojave, 8GB RAM com processador Intel@ QuadCoreMT i5 7g (4 núcleos), CPU 3,4GHz, com buster de $3,8 \mathrm{GHz}$.

Este sistema não apresenta unidades geradoras termoelétricas, porém, é possível simular que uma das unidades seja, isso para fins ilustrativos do método.

Sem considerar restrições de rampa e com manobras no ramo 1-3 entre 3 e 8 horas da manhã, temos o seguinte despacho da Figura 5.

No despacho visualizado na Figura 5, destacamos a unidade geradora 8 por apresentar maior contribuição para demanda. Esta unidade será escolhida para simular uma unidade geradora termoelétrica. Na Figura 6 é possível visualizar as variações sofridas da hora $i$ para a hora $i+1$, supondo que na hora 0 ela já esteja em funcionamento.

No gráfico da Figura 6, algumas variações se destacam, por exemplo, entre as horas 6-7, 18-19 e 22-23, com variações superiores a 5MW, sendo a maior variação entre as horas 18-19. Deste modo, ao inserir restrição de rampa na unidade 8 , para que seja possível identificar as variações nos despachos do sistema, faz-se necessário que o limite de variação máxima de potência entre as horas $i$ e $i+1$ seja inferior a $6 M W / h$, caso contrário, o despacho do sistema IEEE30 não sofrerá alterações.

Com o intuito de provocar alterações no despacho visualizado na Figura 5, serão simuladas rampas de $1 M W / h, 2 M W / h, 3 M W / h, 4 M W / h$ e $5 M W / h$. Além disso, para todas as simulações a função objetivo será apenas minimizar o custo da geração, considerando custos de geração iguais 
entre as unidades, deste modo, não havendo variação de função objetivo entre os cenários. Nestes testes, o foco será apenas no despacho, de modo a facilitar a compreensão do fenômeno que ocorre ao inserir tais restrições de rampa. Deste modo, tem-se os seguintes despachos visualizados nas Figuras 7, 8, 9, 10 e 11.

Comparando com a Figura 5, é possível observar que as restrições de rampa na unidade geradora 8 provocaram variações no despacho, mais perceptíveis na unidade 8 . Pode-se observar que nos intervalos em que a unidade 8 teve seu despacho reduzido, as demais unidades supriram a demanda do sistema tendo seu despacho aumentado por igual. Este comportamento das demais unidades era esperado, uma vez que não houve alteração de demanda.

Observando o despacho da unidade 8 na Figura 5, pode-se ver que há uma grande variação entre as horas 6 e 22. Observando o mesmo intervalo de horas no despacho na Figura 7, pode-se notar que o gráfico da unidade 8 se comporta como uma reta crescente em todo intervalo, uma vez que precisa aumentar sua geração que está limitado a $1 M W / h$.

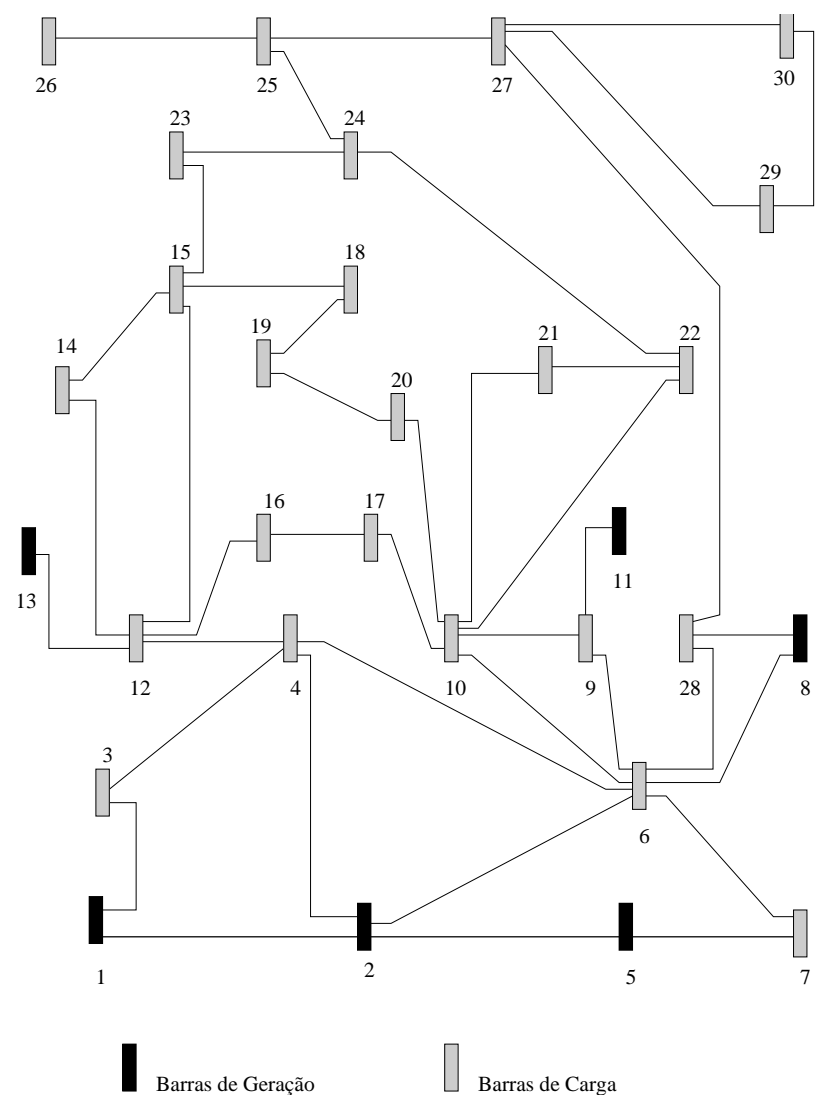

Figura 4: Diagrama Unifilar IEEE30. 


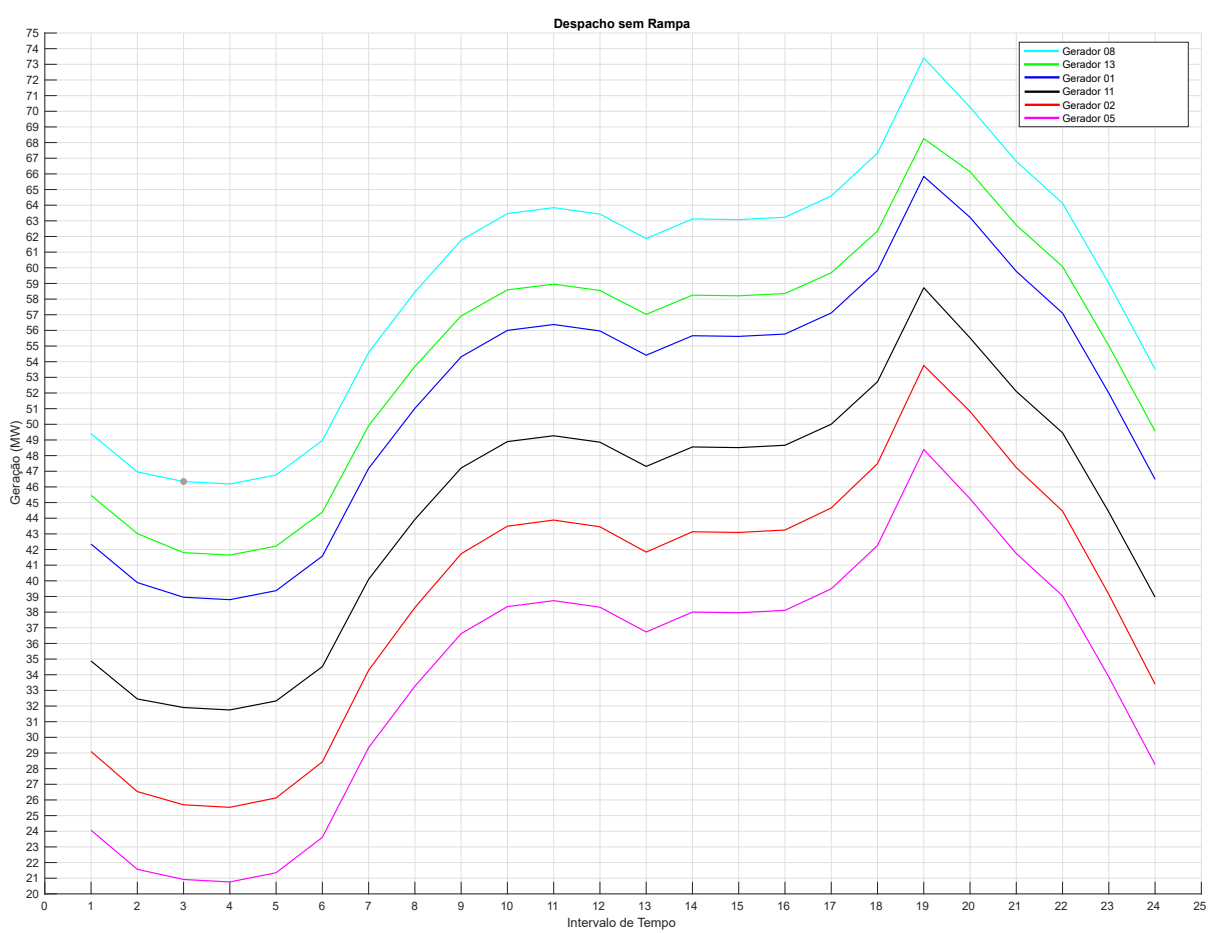

Figura 5: Despacho sem restrição de rampa com número de iterações=3 e tempo computacional $=0.01256$ segundos.

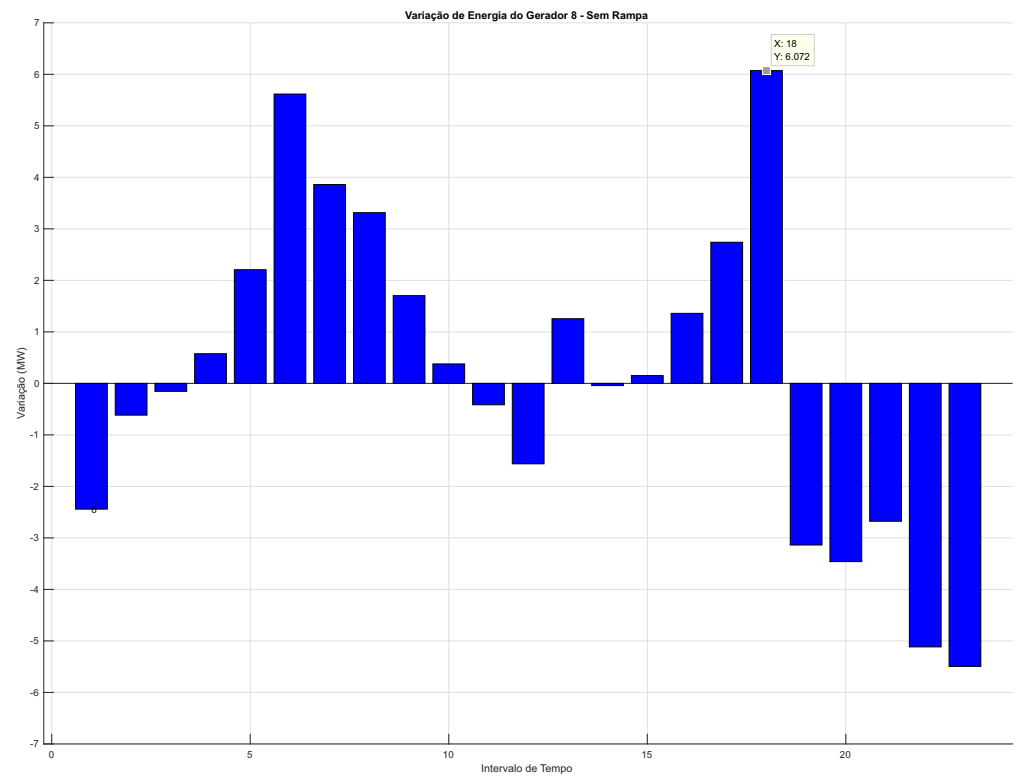

Figura 6: Variação de Potência - Despacho sem restrição de rampa. 


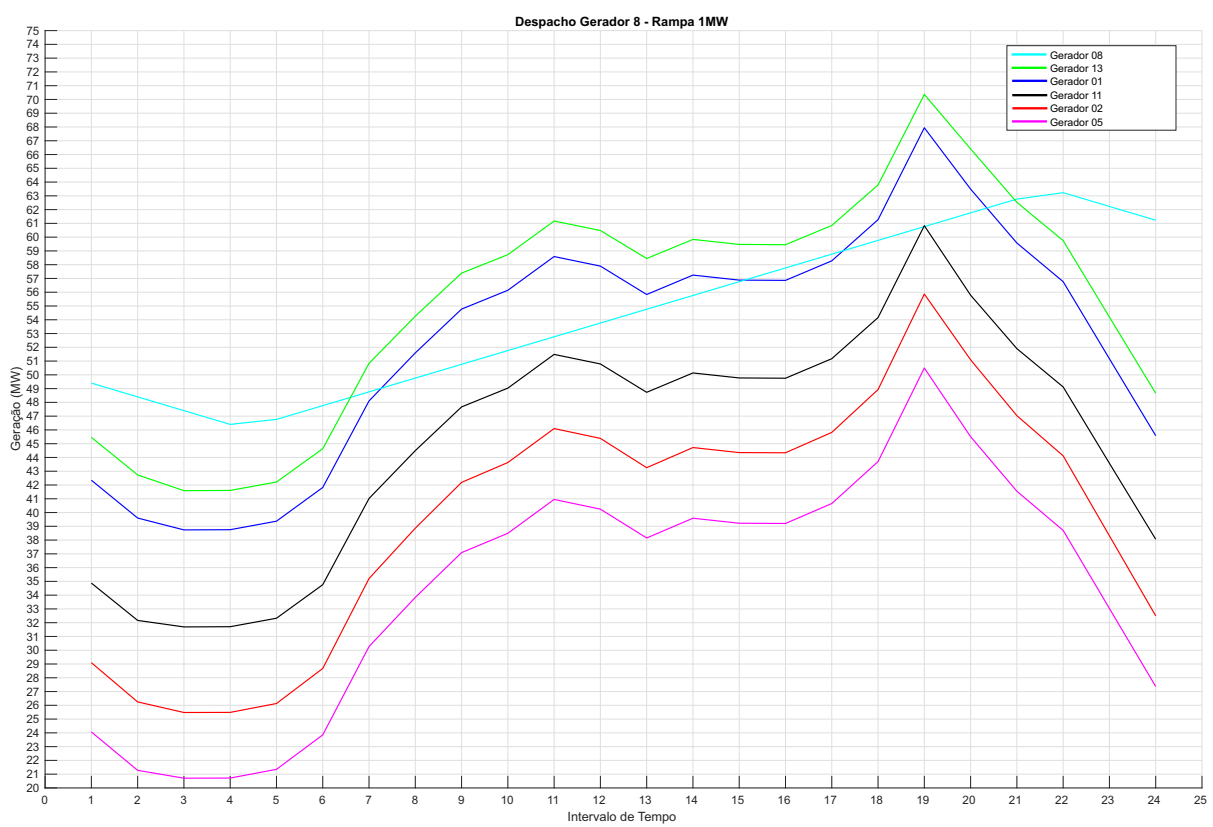

Figura 7: Despacho com restrição de rampa no gerador 8 - 1MW/h com número de iterações=3 e tempo computacional $=0.01298$ segundos.

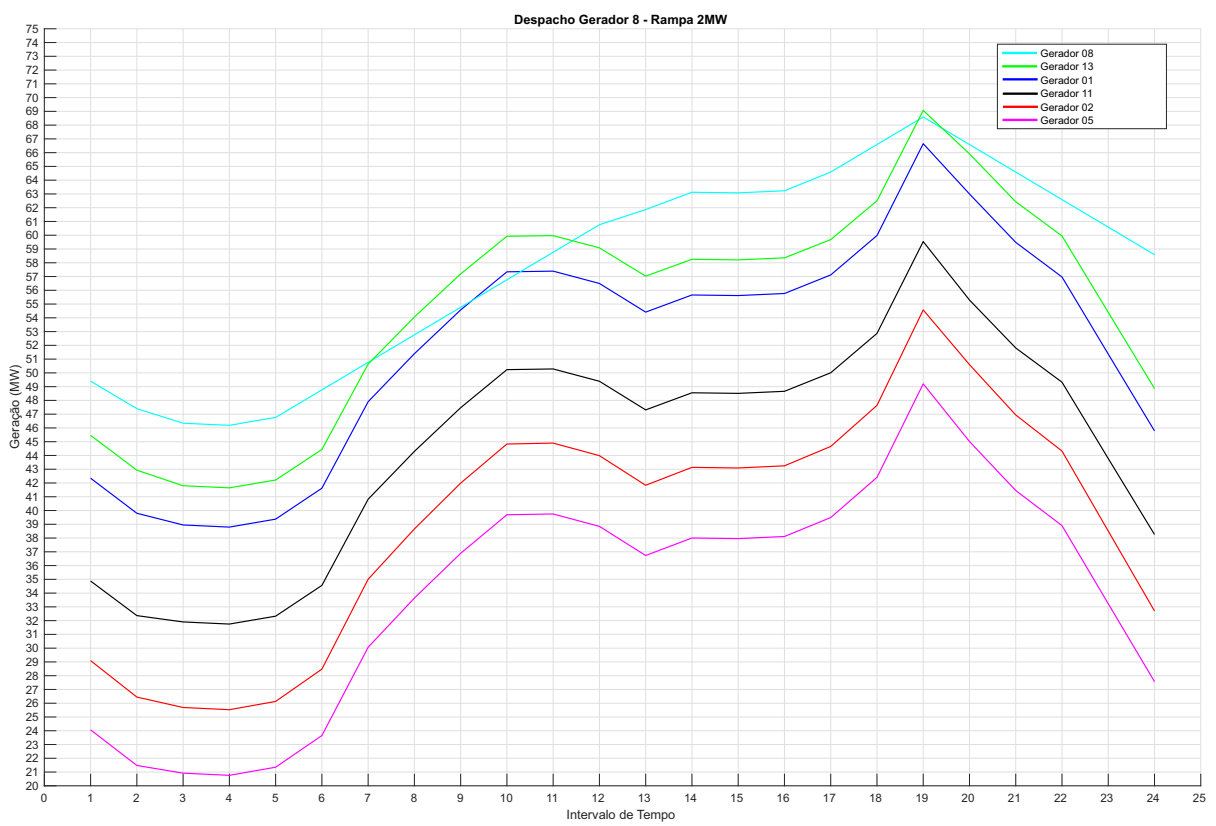

Figura 8: Despacho com restrição de rampa no gerador 8 - 2MW/h com número de iterações=3 e tempo computacional $=0.02239$ segundos. 


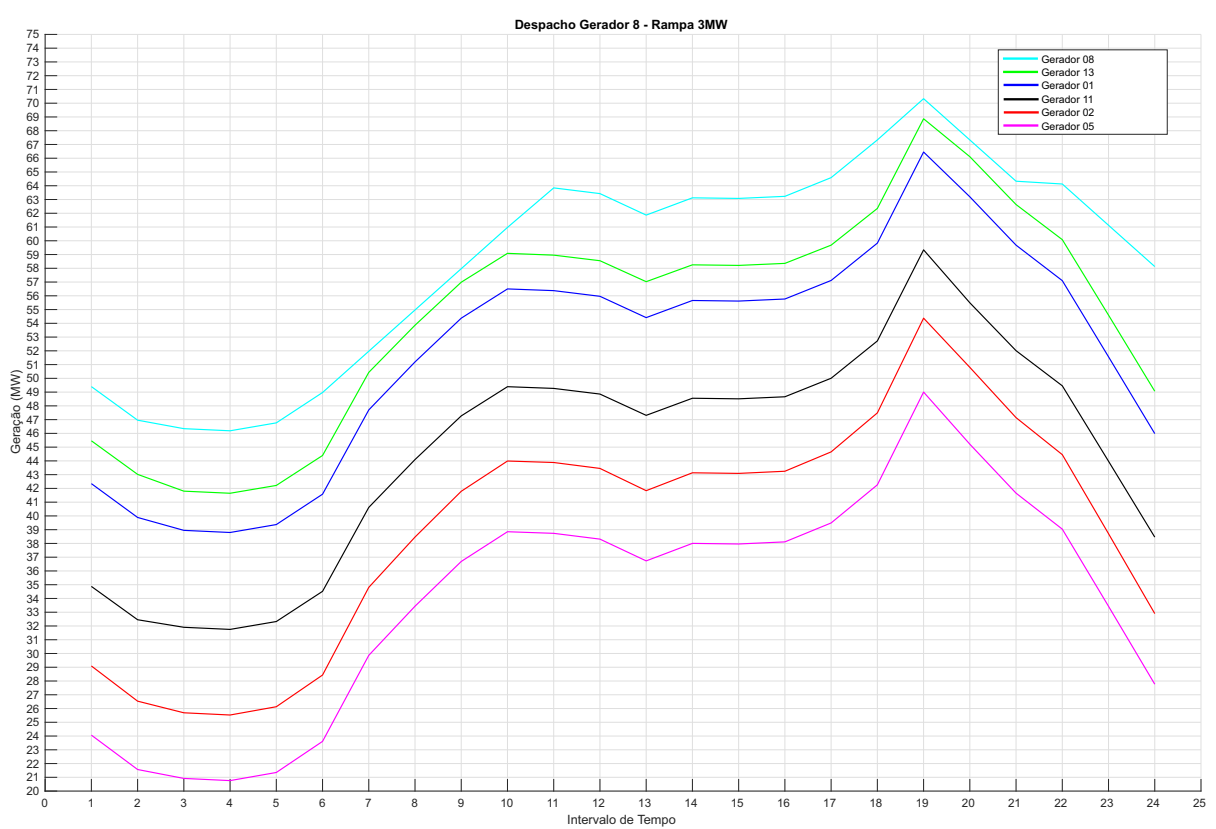

Figura 9: Despacho com restrição de rampa no gerador 8 - 3MW/h com número de iterações=3 e tempo computacional $=0.05277$ segundos.

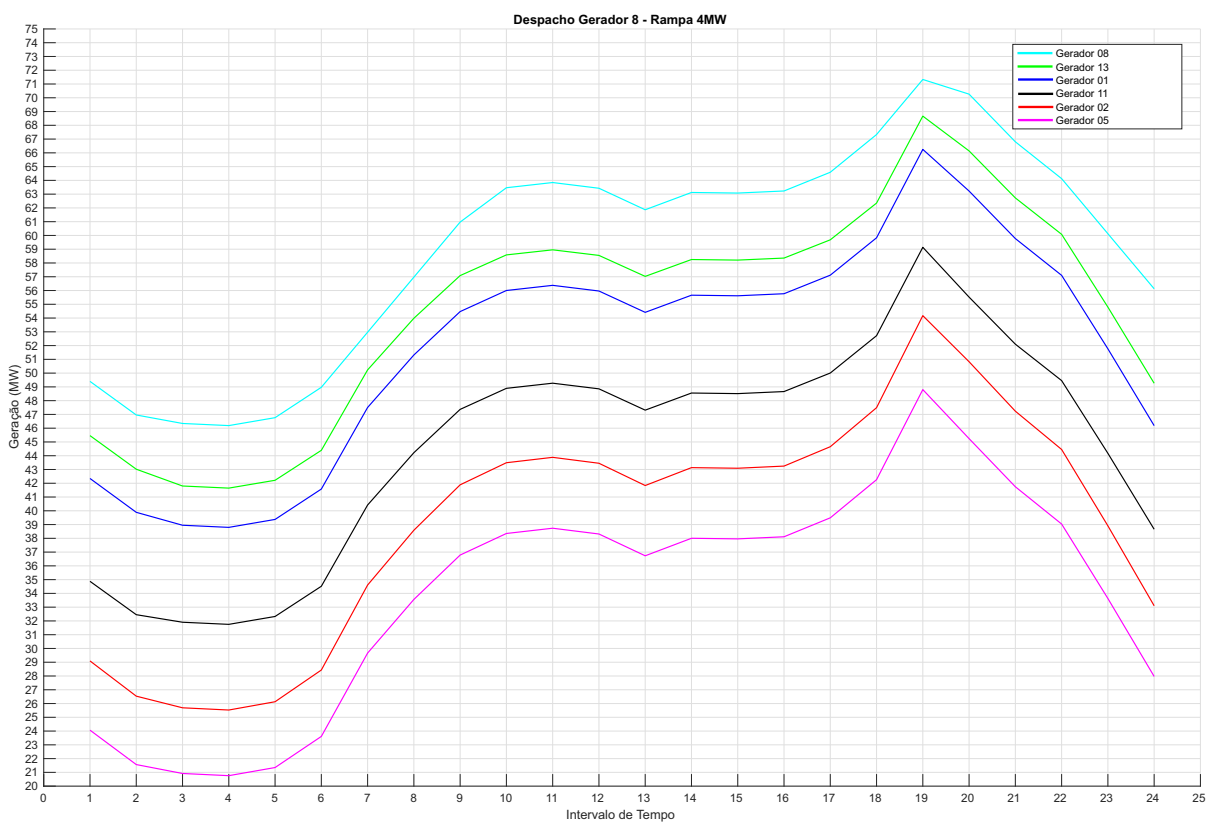

Figura 10: Despacho com restrição de rampa no gerador 8 - 4MW/h com número de iterações=3 e tempo computacional $=0.07856$ segundos . 


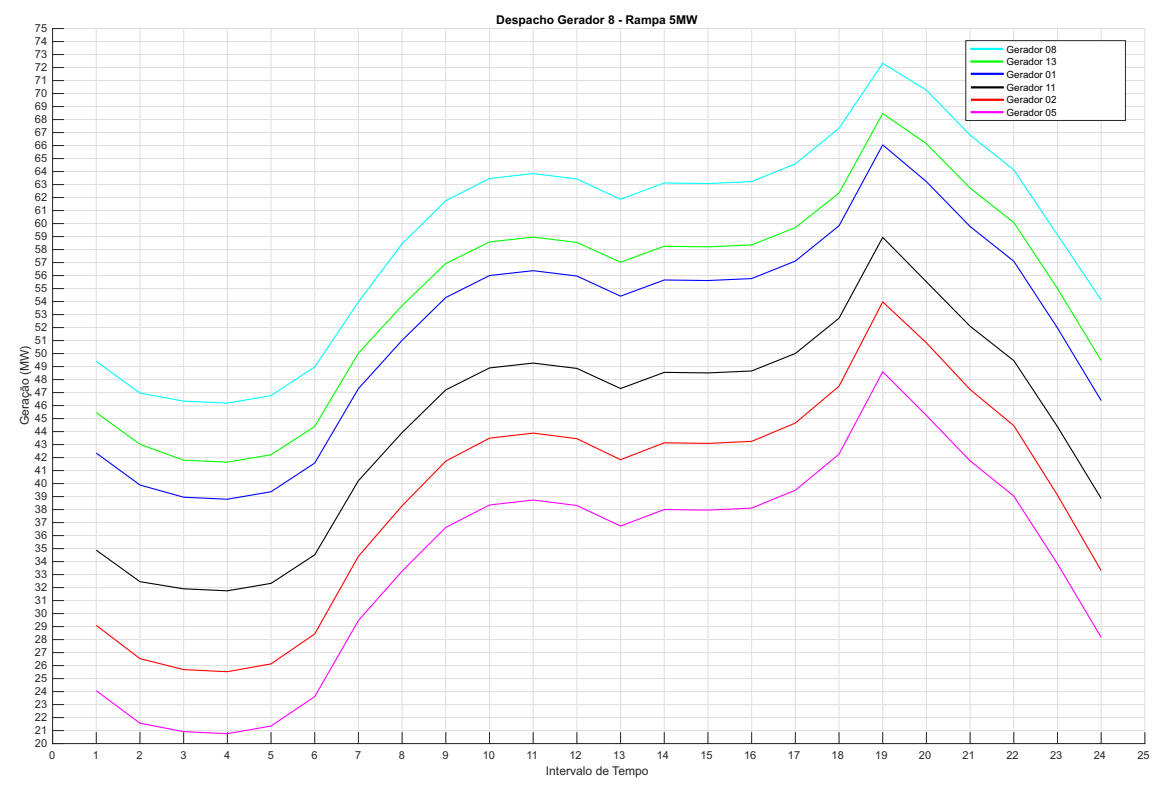

Figura 11: Despacho com restrição de rampa no gerador 8 - 5MW/h com número de iterações=3 e tempo computacional $=0.10331$ segundos.

O gráfico da Figura 12 apresenta uma comparação entre os despachos provocados pelas diferentes restrições de rampa na unidade 8 .

Para ficar mais claro o efeito das restrições de rampa nos despachos da unidade 8 , o gráfico da Figura 13 traz a variação de potência nos cenários propostos.

A Figura 13 ilustra bem a influência da restrição de rampa na unidade geradora, sendo possível observar que em nenhum momento esta restrição foi violada.

Entendendo os comportamentos citados neste artigo, foi possível simplificar diversos pontos da implementação, além de observar outros fenômenos como por exemplo, a variação dos multiplicadores de lagrange, que podem indicar a "necessidade"de maior liberdade de rampa, reduzindo a cada aumento de limite a variação de potência.

\section{CONCLUSÕES}

Os resultados obtidos relacionados ao pré-despacho confirmam a idéia que métodos de pontos interiores especializados a estruturas particulares podem levar a implementações que obtém bons resultados computacionais em termos de tempo. A robustez obtida em todas as implementações é um resultado esperado, típico destes métodos na área de sistemas de potência. A modelagem de problemas cada vez mais próximas do problema real operado pelo ONS permite o desenvolvimento futuro de uma ferramenta que poderá ser utilizada na operação do sistema interligado nacional. 


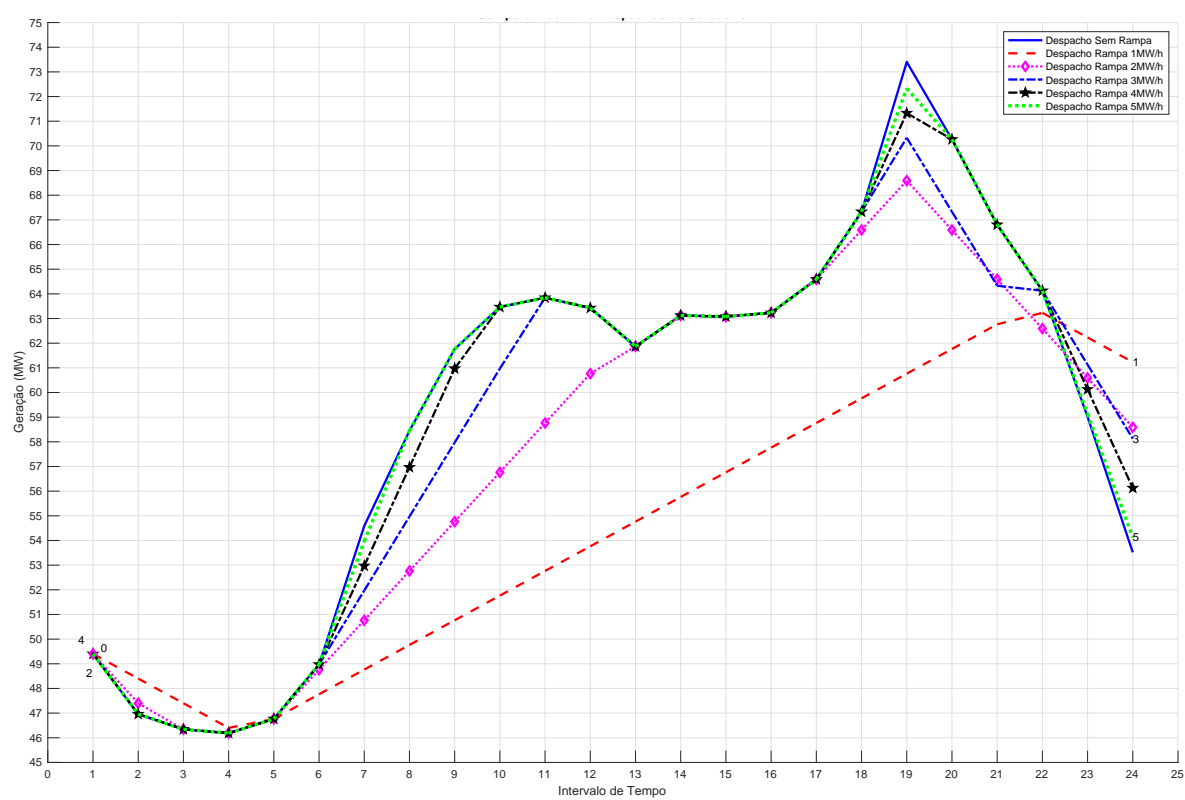

Figura 12: Despachos com restrição de rampa no gerador 8.

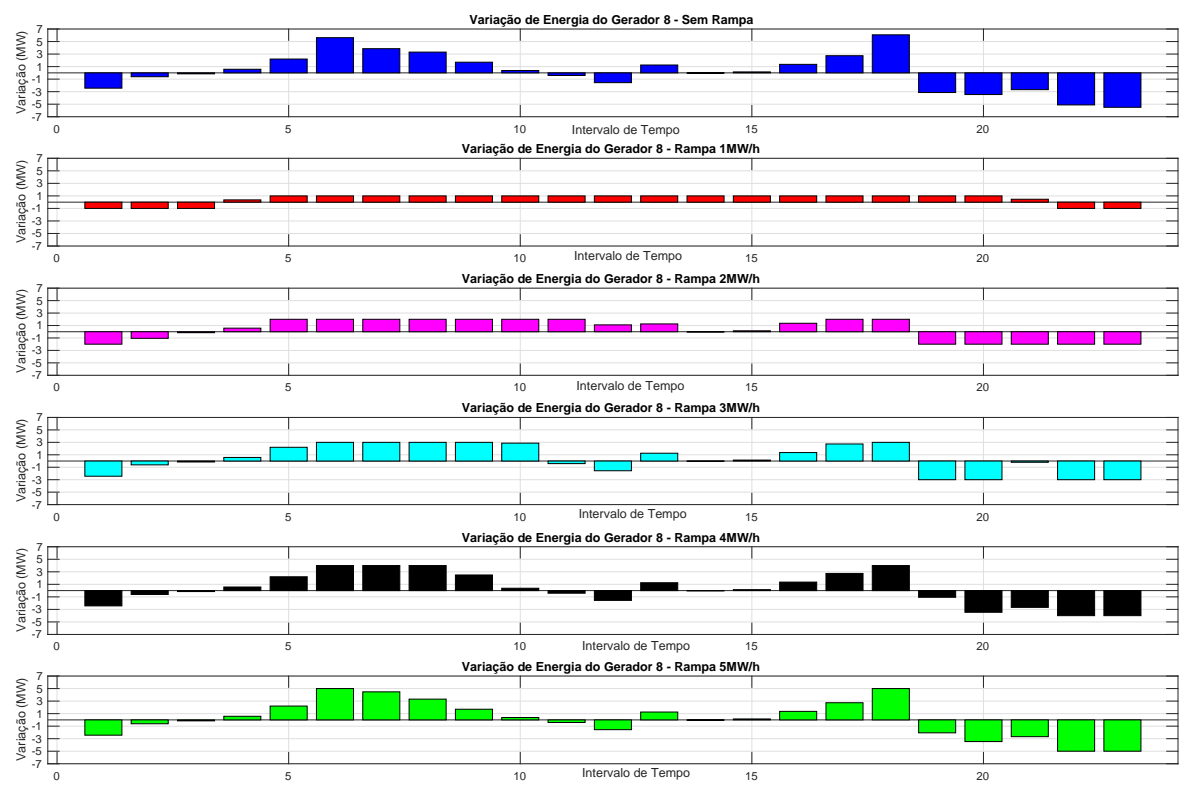

Figura 13: Variação de Potência da unidade 8 para cada restrição de rampa. 
Vale destacar que restrições de rampa em todas as unidades geradores pode ser inviável dependendo da demanda, uma vez que foi observado que se uma unidade com restrição de rampa reduz seu despacho em comparação ao despacho original, então alguma unidade deverá suprir a necessidade do sistema.

\begin{abstract}
The primal-dual interior point methods will be used for the problem of loss minimization in the generation predispatch of a hydroelectric and hydrothermal power system where further programmed maneuvers and ramp restrictions occur. With the variation of demand, it is necessary to carry out some scheduled maintenance for generators and to adapt the transmission network to this load, enabling it to efficiently and keeping the system stable. The thermoelectric plants, in turn, present ramp restrictions, since they need a certain time to increase as well as to reduce their energy production. The consideration of additional maneuvers and restrictions approximates the model of the predispatch problem of the Brazilian system according to the National System Operator.
\end{abstract}

Keywords: interior points methods, ramp constraints, programmed maneuvers.

\title{
REFERÊNCIAS
}

[1] I. Adler, M.G.C. Resende, G. Veiga \& N. Karmarkar. An Implementation of Karmarkar's Algorithm for Linear Programming. Mathematical Programming, 44 (1989), 297-335.

[2] R. Ahuja, T. Magnanti \& J.B. Orlin. "Network Flows”. Prentice Hall, United States (1993).

[3] S. Bocanegra, F.F. Campos \& A.R.L. Oliveira. Using a Hybrid Preconditioner for Solving Large-Scale Linear Systems arising from Interior Point Methods. Computational Optimization and Applications, (2007), 149-164.

[4] M.F. Carvalho, S. Soares \& T. Ohishi. Optimal Active Power Dispatch by Network Flow Approach. IEEE Transactions on Power Systems, 3(3) (1988), 1640-1647.

[5] S.M.S. Carvalho. "Métodos de pontos interiores aplicados ao pré-despacho com manobras simultâneas de barras e linhas". Ph.D. thesis (2010). Tese doutorado.

[6] S.M.S. Carvalho \& A.R.L. Oliveira. Interior Point Methods Applied to the Predispatch Hydroelectric System with Simulated Modification in the Network Topology. IEEE Latin America Transactions, 13 (2015), 143-149.

[7] J. Castro. A Specialized Interior-Point Algorithm for Multcommodity Network Flows. SIAM J. Optimization, 10(3) (2000), 852-877.

[8] J. Czyzyk, S. Mehrotra, M. Wagner \& S.J. Wright. PCx An Interior Point Code for Linear Programming. Optimization Methods \& Software, 11-2(1-4) (1999), 397-430.

[9] P. Franco, M.F. Carvalho \& S. Soares. A Network Flow Model for Short-Term Hydro-Dominated Hydrothermal Scheduling Problem. IEEE Transactions on Power Systems, 9(2) (1994), 1016-1021.

[10] A. Garzillo, M. Innorta \& R. Ricci. The flexibility of interior point based power flow algorithms facing critical network situations. Electrical Power \& Energy Systems, 21 (1999), 579-584. 
[11] J. Gondzio. Multiple Centrality Corrections in a Primal-Dual Method for Linear Programming. Computational Optimization and Applications, 6 (1996), 137-156.

[12] J.L. Kennington \& R.V. Helgason. "Algorithms for Network Programming”. Wiley, New York (1980).

[13] I.J. Lustig, R.E. Marsten \& D.F. Shanno. On Implementing Mehrotra's Predictor-Corrector Interior Point Method for Linear Programming. SIAM Journal on Optimization, 2 (1992), 435-449.

[14] C. Lyra, S.M.S. Carvalho \& A.R.L. Oliveira. Predispatch of hydroelectric power systems with modifications in network topologies. Annals of Operations Research - Springer, 1 (2018), 1-19.

[15] J.A. Momoh, M.E. El-Hawary \& R. Adapa. A Review of Selected Optimal Power Flow Literature to 1993, Part II Newton, Linear Programming and Interior Point Methods. IEEE Transactions on Power Systems, 14(1) (1999), 105-111.

[16] A.R.L. Oliveira \& A.M. Lima. Comparação Entre Diferentes Formulações do Problema de Fluxo de Potência Ótimo Utilizando o Método de Pontos Interiores. Tendências em Matemática Aplicada e Computacional, 5 (2004), 267-279.

[17] A.R.L. Oliveira \& C. Lyra. Interior Point Methods for the Polynomial $L_{\infty}$ Fitting Problem. International Transactions in Operational Research, 11(3) (2004), 309-322.

[18] A.R.L. Oliveira, M.A. Nascimento \& C. Lyra. Efficient Implementation and Benchmark of Interior Point Methods for the Polynomial $L_{1}$ Fitting Problem. Computational Statistics \& Data Analysis, 35(2) (2000), 119-135.

[19] A.R.L. Oliveira \& R.W. Probst. Aplicação dos Métodos de Pontos Interiores Primais-Duais ao Problema de Pré-despacho de um Sistema Hidrotérmico. Anais do XXXVII Simpósio Brasileiro de Pesquisa Operacional, (2005), 1900-1908.

[20] A.R.L. Oliveira \& S. Soares. Métodos de Pontos Interiores para Problema de Fluxo de Potência ótimo DC. SBA: Controle \& Automação, 14(3) (2003), 278-285.

[21] A.R.L. Oliveira, S. Soares \& L. Nepomuceno. Optimal Active Power Dispatch Combining Network Flow and Interior Point Approaches. IEEE Transactions on Power Systems, 18(4) (2003), 1235-1240.

[22] A.R.L. Oliveira, S. Soares \& L. Nepomuceno. Short Term Hydroelectric Scheduling Combining Network Flow and Interior Point Approaches. Electrical Power \& Energy Systems, 27(2) (2005), 91-99.

[23] A.R.L. Oliveira \& D.C. Sorensen. A New Class of Preconditioners for Large-Scale Linear Systems from Interior Point Methods for Linear Programming. Linear Algebra and Its Applications, 394 (2005), 1-24.

[24] V.H. Quintana, G.L. Torres \& J.M. Palomo. Interior Point Methods and their Applications to Power Systems: A Classification of Publications and Software Codes. IEEE Transactions on Power Systems, 15(1) (2000), 170-176. 
[25] M. Resende \& G. Veiga. An efficient implementation of a network interior point method. In D. Johnson \& C. McGeoch (editors), "Network Flows and Matching: First DIMACS Implementation Challenge. DIMACS Series on Discrete Mathematics and Theoretical Computer Science", volume 12 (1993), pp. 299-348.

[26] R.J. Vanderbei. "Linear Programming - Foundations and Extensions". Kluwer Academics Publishers, Boston, USA (1996).

[27] S.J. Wright. "Primal-Dual Interior-Point Methods". SIAM Publications, SIAM, Philadelphia, PA, USA (1996). 